\title{
Artificial neural network simulating microbial fuel cells with different membrane materials and electrode configurations.
}

\author{
Michail-Antisthenis Tsompanas ${ }^{\mathrm{a}}$, Jiseon You ${ }^{\mathrm{a}}$, Lauren Wallis ${ }^{\mathrm{a}}$, John Greenman ${ }^{\mathrm{a}}$, Ioannis Ieropoulos ${ }^{\mathrm{a}}$ \\ ${ }^{a}$ Bristol BioEnergy Centre, University of the West of England, Bristol BS16 1QY, UK
}

\begin{abstract}
Microbial fuel cells (MFCs) are gaining interest due to higher power production achieved by deep analysis of their characteristics and their effect on the overall efficiency. To date, investigations on MFC efficiency, can only be based on laboratory experiments or mathematical modelling. However, there is only a handful of rule-based mathematical modelling due to the difficulties imposed by the high sensitivity of the MFC system to environmental parameters and the highly complex bacterial consortia that dictate its behavior. Thus, an application of an artificial neural network (ANN) is proposed to simulate the polarisation of cylindrical MFCs with different materials as the separation membranes. ANNs are ideal candidates for investigating these systems, as there is no need for explicit knowledge of the detailed rules that govern the system. The ANN developed here is a feed-forward back-propagation network with a topology of 4-10-1 neurons that approximates the voltage of each MFC at a given state. Two different membrane materials with two different electrode configurations were assembled and utilized in laboratory experiments to produce the data set on which the ANN was trained upon. For the whole data set the correlation coefficient $(R)$ between real values and outputs of the network was 0.99662 .
\end{abstract}

Keywords: Artificial neural networks, microbial fuel cells, polarisation curves

\section{Introduction}

The ability of Microbial Fuel Cells (MFCs) to extract electricity while processing waste-water has brought them in the spotlight of many researchers' interest. This is achieved by the employment of the metabolism of a microbial biofilm as an oxidation agent. A MFC is divided into two compartments, the anode and the cathode, by a membrane that allows the movement of positively charged ions. The biofilm is attached on the anode electrode and the bacteria that constitute it produce electrons and positively charged ions, as part of their metabolism. The electrons flow through the anode electrode through an external circuit to the cathode electrode, while the positively charged ions diffuse through the membrane to the cathode department. Positively charged ions and electrons merge with oxygen in the cathode electrode to fulfill the reduction-oxidation reaction cycle. 
The task of optimizing MFC technology is heavily based on laboratory tests [1, 2, 3, that usually involve changing one parameter at a time. However, due to high costs and long time intervals required in laboratory tests, mathematical modelling and simulated optimisation of MFCs has been proposed as a viable alternative 4, 5, 6, 7, 8, 9, 10, 11, even though such modelling is subject to some level of abstraction.

Additionally, some parameters that are critical for the efficiency of MFCs are difficult to control or even measure in situ. Some of these parameters are the biofilm thickness on the anode electrode, the actual composition of the bacteria consortium in the biofilm, their growth rate and substrate utilization rate [12, 13, 14, 15, 16]. An alternative that can alleviate these difficulties is modelling MFCs with machine learning techniques. In this way only system inputs and outputs are required, while the rules governing the system will be unveiled by the algorithm, without any prior high-level knowledge required of the system in question.

Consequently, there is a current trend in using (artificial neural networks) machine learning solutions in modelling and predicting the performance of MFCs. Using ANN does not involve developing an ensemble of highly accurate formulas that describe all processes (physical, biological and electro-chemical) that occur within the system. On the contrary, only input and output data are required and the ANN uncovers in an automatic way the relations between inputs and outputs. Thus, no explicit knowledge of the system is required (however, expertise on the field on MFC is required to help selecting and using the meaningful inputs that have effects on the outputs).

The field of artificial neural networks (ANNs) is highly inspired by the way that the human brain functions. The motivation derives from the fact that the brain computes in a completely different method than well-established digital computers. While digital computers are developed in a highly organized manner with modules that perform specific and predefined functions, the brain works in a nonlinear and parallel manner with high complexity. The advantages of ANNs that are simplified models of the way parts of the human brain work, are their parallel distributed structure as well as the ability to learn and, therefore, generalize. Generalization is defined as the ability to produce good estimated outputs for inputs that were not processed during the learning function of the network.

In the present study a cylindrical type of MFCs was studied. Two different materials for membrane were used. Moreover, different configurations of the anode and cathode compartments were investigated. One with the anode on the outside (cathode on the inside) and one with the anode on the inside (cathode on the outside). The four test cases were run in triplicate, thus resulting in 12 MFCs.

A forward-fed and back-propagation ANN with topology of 4-10-1 neurons was developed. The four input neurons represent the four input parameters and the output neuron, the voltage of the MFC. The data set that was produced by the laboratory experiments, consisted of 264 samples $(12$ MFCs $\times 22$ values of load resistances). The correlation coefficient $(R)$ between real values and outputs of the network was 0.99662 for all the values in the data set (training, validation and testing fragments). 


\section{Previous Work}

Recognizing the difficulties of designing a detailed rule-based model to simulate the outputs of a MFC there were several attempts to predict the output of a system with an ANN. In [17] a fully connected multi-layer perceptron ANN [forward-fed and back-propagation neural network (FFBPNN)] was developed to approximate the current output of MFCs. Inputs were $\mathrm{pH}$, biological oxygen demand, chemical oxygen demand and total suspended solids (TSS). The data set used was extracted from previously published investigations that involved functionality of MFCs fed with several types of waste-water, like brewery waste, sugar industry, dairy wastewater, municipal wastewater and waste from the paper industry.

Moreover, the authors of [18] approximated with an ANN the outputs of a membrane-less MFC that they fabricated. The inputs for the network were temperature, $\mathrm{pH}$ and electron acceptor concentration, whereas power and current density were selected as the outputs. By using different amounts of hidden layer neurons, the best results were produced with three neurons. The coefficient of determination $\left(R^{2}\right)$ reported for this architecture was 0.9886 for the testing data set.

Nonetheless, additional approaches of artificial intelligence (AI) were tested (like multi-gene genetic programming (MGGP) and support vector regression) in comparison with ANN for the approximation of the MFC outputs [19]. In this previous study [19] only two input parameters were used, namely temperature and ferrous sulfate concentrations. The goal was to predict the output voltage during two different functionality periods of the system, before and after start-up. The data set used was extracted from previously published investigations. The MGGP method proved as the most efficient one, providing coefficient of determination $\left(R^{2}\right)$ as high as 0.9872 , while the ANN as high as 0.9588 for a testing fragment of the data set.

Another algorithm that ANNs were compared with in the context of MFC modelling, was adaptive neurofuzzy inference system (ANFIS) [20. The authors of that study defined as inputs the ionic strength, initial $\mathrm{pH}$, medium nitrogen concentration and temperature, whereas the outputs were power density and Columbic efficiency. The data set used was once more derived by previously published results. The conclusion drawn was that while both approaches provide very accurate estimations (indicated with several measures like average relative error, absolute average relative error, standard deviation and the correlation coefficients), the ANN approach is simpler to implement

The authors of 21] used an ANN to simulate the power generation in a MFC, considering the anode electrode positioning and the flow rate. They set up laboratory experiments of MFCs with altering the angle of the anode electrode at 0,45 and $90^{\circ}$ with respect to the flow direction and the flow rate at 1 and $2 \mathrm{~mL} / \mathrm{min}$. These parameters along with time were used as inputs, whereas the output of the network was the power generated. The results from the trained network were in good agreement with the measured ones, showing a coefficient of determination of 0.99944 .

Moreover, the loading of common fuel for MFCs, such as domestic wastewater, with powdered giant 
reed, was studied and approximated by an ANN [22]. The network was trained with the data acquired by laboratory runs with different concentrations of giant reed and particle sizes. The output of the network was defined as the power density of the MFC and the inputs as the duration in days, the concentration of giant reed in wastewater and its particle sizes. Several numbers of neuron in the hidden layer were tested, with the best performance reached with 12 neurons with a coefficient of determination of 0.9993 .

In 223] the performance of $33 \mathrm{MFCs}$ were examined in terms of power density, Coulombic efficiency and COD removal rates. The MFCs were subject to different conditions, such as conductivity, nitrogen, phosphorus concentrations, substrate type and substrate concentration. They were fed with eight separate substrates (acetate, butyrate, fumarate, glucose, fructose, galactitol, bovine serum albumin, diesel range hydrocarbon mixture) and three different types of wastewater (brewery, potato processing and dried fruit processing). To predict the aforementioned three measures of performance, three ANNs with different configurations were trained upon the data extracted. The first kind of network utilized the wastewater/solution parameters to project the taxon composition and then both for the performance parameters. The second kind of network is like the first kind but uses only the predicted taxonomy to provide the performance parameters. While the third kind uses only wastewater/solution parameters to predict both taxonomy and performance. According to the authors, the motivation of the different types of ANNs was the attempt to extract data associations between biofilm composition, reactor outputs and wastewater compositions. The first kind of ANN proved to be more efficient in calculating Coulombic efficiency and COD removal rates.

Additionally, other types of bioreactor were studied with ANNs. For instance, the bio-hydrogen yield in microbial electrolysis cells (MECs) was modelled with ANNs equipped with a different number of neurons in the hidden layer 24. The inputs were defined as substrate type, substrate concentration, $\mathrm{pH}$, temperature, applied voltage and reactor configuration. The data set used was once more derived from previously published work. The most accurate result, providing a coefficient of determination $\left(R^{2}\right)$ of 0.90 , was achieved with six neurons in the hidden layer.

Finally, more data-driven models studying MFCs were presented using different approaches, like a nonparametric Gaussian process regression (GPR) model [25] and a support vector regression forward and inverse model [26].

Drawing inspiration from the aforementioned studies we utilised the ANN methodology to simulate the polarisation curves of MFCs with different membrane material and configurations of electrodes. No previous study using ANNs was approximating polarisation curves. Moreover, the load resistance was not used in any of the previous published works as an input parameter of the network. Nonetheless, despite the fact that the majority of previous work use small data sets (from 17 to 200 data samples) with unique input-output pair values, in this study the produced data set, that is used to train the network, is more extensive (i.e. 264 data samples) and contains more than one instance for the same set of inputs. The result is a more accurate model of the polarisation behaviour, which successfully simulates even the overshoot phenomenon, 
which has not been previously demonstrated in MFC modelling papers.

\section{Methods}

Two types of membranes with different materials were used for building the MFCs under study. One type was terracotta cylinders (Weston Mill Pottery, Nottinghamshire, UK) of $7 \mathrm{~cm}$ length, with $1.5 \mathrm{~cm}$ inner diameter and wall thickness of $1 \mathrm{~mm}$. The other type was brown-black clay cylinder (Goerg \& Schneider, Siershahn, Germany) of $7 \mathrm{~cm}$ length, with $1.7 \mathrm{~cm}$ inner diameter and wall thickness of $2 \mathrm{~mm}$. The cylinders were placed in cylindrical plastic housing to provide the anode or cathode compartment, with a maximum fuel capacity of $60 \mathrm{ml}$. The anode electrode was a layer of carbon fibre veil (carbon loading $20 \mathrm{gm}^{-2}$ ) in a rectangular shape with a total macro surface area of $270 \mathrm{~cm}^{2}$ (PRF Composite Materials, Dorset, UK), folded and wrapped around the outside or placed inside the cylindrical membranes and held with stainless steel wire, which was also equipped as a current collector and the connection point. Open-to-air type cathodes made of heat-pressed activated carbon onto the carbon fibre veil were used. MFCs were inoculated with 1:1 mixture of human urine and activated sewage sludge (Wessex Water Scientific Laboratory, Cam Valley, Saltford, UK) enriched with $1 \%$ tryptone, $0.5 \%$ yeast extract and $0.5 \%$ sodium acetate. The MFCs were fed with human urine continuously at a flow rate of $14 \mathrm{mLh} \mathrm{h}^{-1}$ and initially loaded with an external resistance of $500 \Omega$. The anode of each MFC was matured for 12 days, prior to starting the polarisation curve experiments.

The behaviour of MFCs exhibited during polarisation tests was investigated here. These tests were executed within several hours (i.e. c. 3 hours for 12 values of resistance) and some key parameters of MFC performance can be considered constant. Thus, they were not included as ANN inputs. One of these parameters is temperature, given the thermostatic wetlab environment.

A standard type of ANN for function fitting was selected, namely a two-layer feed-forward backpropagation neural network. Forward feeding describes the way an output is calculated, whereas, backpropagation is the training algorithm of the network. The neurons are completely connected. There is one hidden layer with neurons implementing a sigmoid transfer function and one output node implementing a linear transfer function.

The aforementioned type of ANN is formed by a predefined finite amount of successive layers. Every layer is accommodating a finite number of neurons (the elementary entity of a neural network). All neurons on a layer are connected to all the neurons on the subsequent layer, and also the previous one. The connections are also known as synapses. The data are fed from one layer to the other through the synapses and the neurons, in a forward manner (from the input layer to the output, as in Fig. 1), this is why this ANN is named feed-forward [27].

More specifically, every neuron in the input layer has an output (same as the input) value denoted as $x_{0 j}$. 


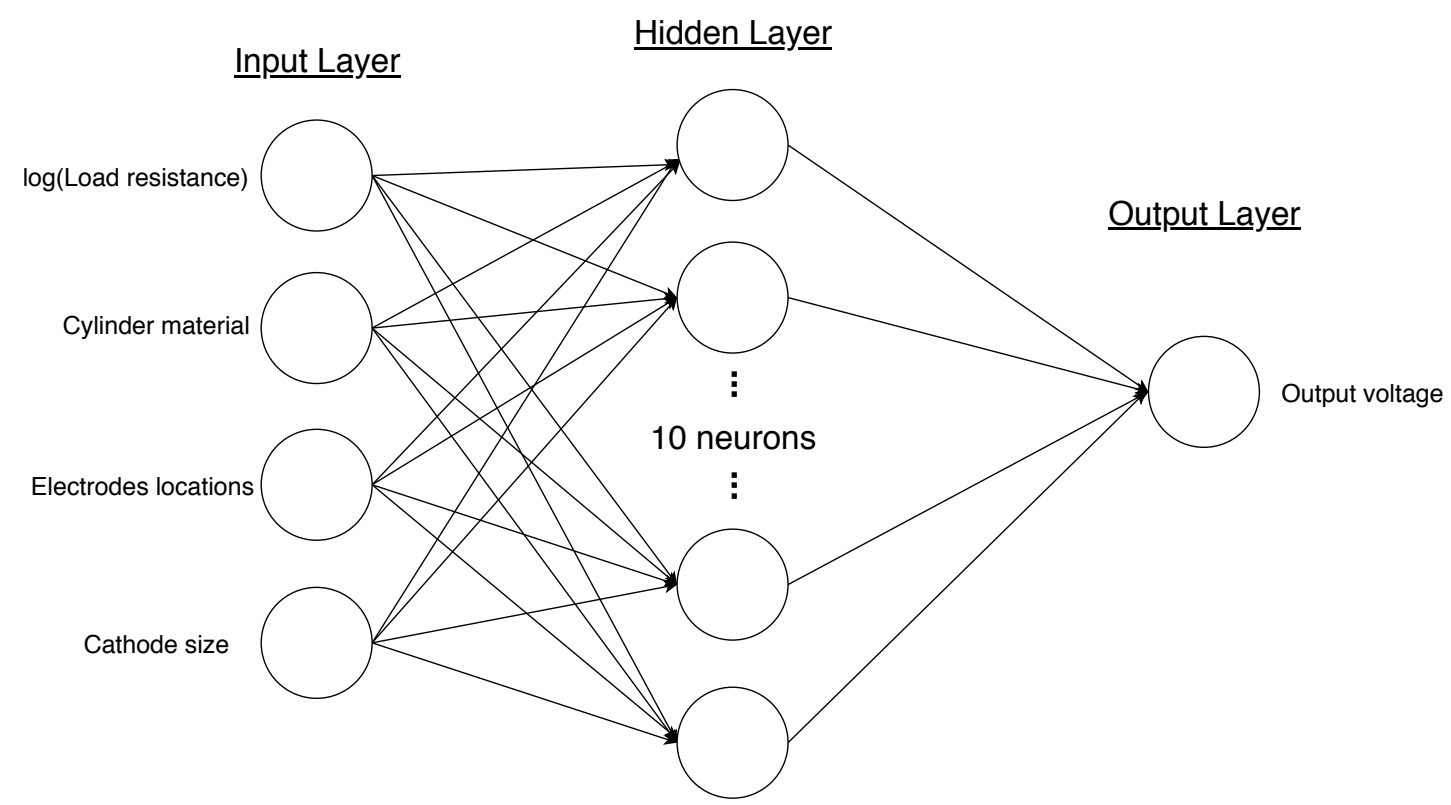

Figure 1: Topology of ANN (with one output).

Then, the $k$ th neuron in the $i$ th layer receives the output from each $j$ th neuron of the $(i-1)$ th layer, denoted as $x_{i j}$, multiplied by the weight of the synapses, denoted as $w_{i j k}$. All the products are added within each neuron, a bias value is subtracted from the sum and the result is applied to the neuron's transfer function. The output of the $k$ th neuron routed towards the $(i+1)$ th layer is:

$$
x_{i+1, k}=\sigma\left(\sum_{j} w_{i j k} x_{i j}-\theta_{i k}\right)
$$

where $\sigma$ is a predetermined transfer function. Here the sigmoid function was used for the hidden layer:

$$
\sigma(\xi)=\frac{1}{1+e^{-\xi}}
$$

The procedure that determines the values of the weights of the synapses is known as training. A common method of training is the back-propagation, which is a gradient descent method. As this procedure is complicated and out of the scope of the present work it will not be explained in detail, but the reader can refer to numerous works that explain in depth the well-established training procedure of ANN [28, 29].

The topology of the network is depicted in Fig. 1. The input nodes represent the input parameters, i.e. logarithmic value of load resistance, cylinder material, electrode location and cathode electrode size. The hidden layer consisted of ten neurons for the initially developed network, while this number was altered to find the most efficient predicting topology. The output neuron represents the MFC's voltage output.

The data set acquired by the aforementioned laboratory experiments, comprised 264 samples (12 MFCs $\times 22$ values of load resistance). For the training procedure, the data set was randomly divided in $70 \%$ (184 
samples) for the training set, 15\% (40 samples) for the validation set and 15\% (40 samples) for the test set. The Levenberg-Marquardt algorithm was used for training the network.

\section{Results}
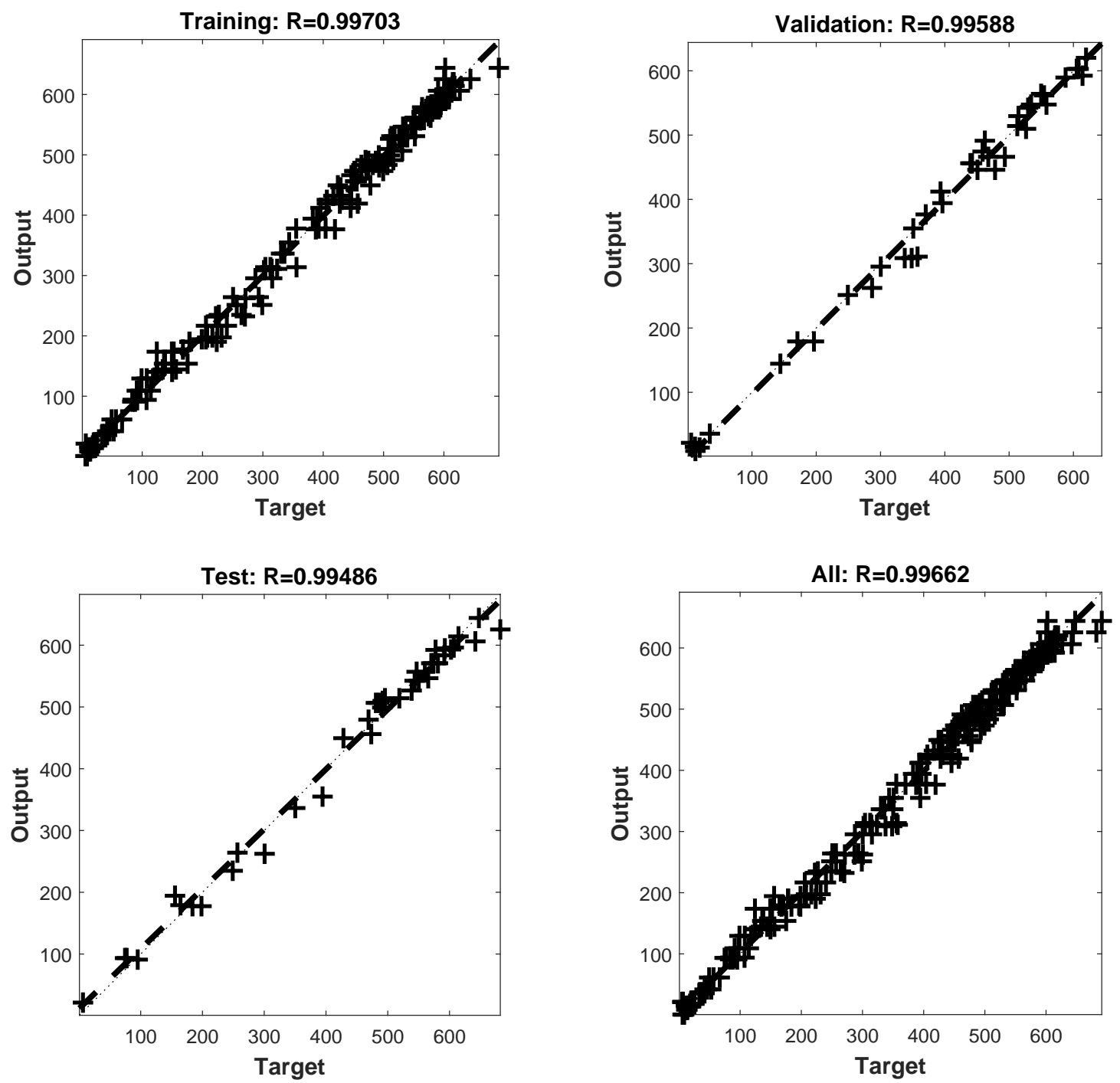

Figure 2: Regression plots showing network results compared with targets for (A) training, (B) validation, (C) test and (D) whole data set.

The regression plots illustrated in Fig. 2 indicate the accuracy of the ANN. The majority of the value points are adequately close to the $45^{\circ}$ line (which indicates a perfect fit of the modelled data to the real data). The correlation coefficient $(R)$ values are $0.99703,0.99588,0.99486$ and 0.99662 for the training, validation, test and whole data set. 
After the network was trained and tested, it was used to produce data (here voltage), for the cases present in the training data set, for illustrating reasons of its outputs. The following figures present the polarisation curves (Figs. 36(a)) and the voltage-resistance curves (Figs. 36(b)) for every different type of MFCs. Note that the measured values (represented as blue diamonds) as well as the simulated values (represented as black circles) are shown for each case. The current values for the polarisation curves were calculated with Ohm's law, using the predicted voltage and the given resistance. Moreover, the red dashed lines (in Figs. 36 6 (b)) represent the distance of one standard deviation from the average mathematical value of the measured voltages for every type of MFCs.

Namely, in Fig. 3 the outputs of the ANN are shown for the case of MFCs (T_Cout) with terracotta membrane, cathode $\left(35 \mathrm{~cm}^{2}\right)$ on the outside of the ceramic cylinder (at the same values of resistances used in lab).

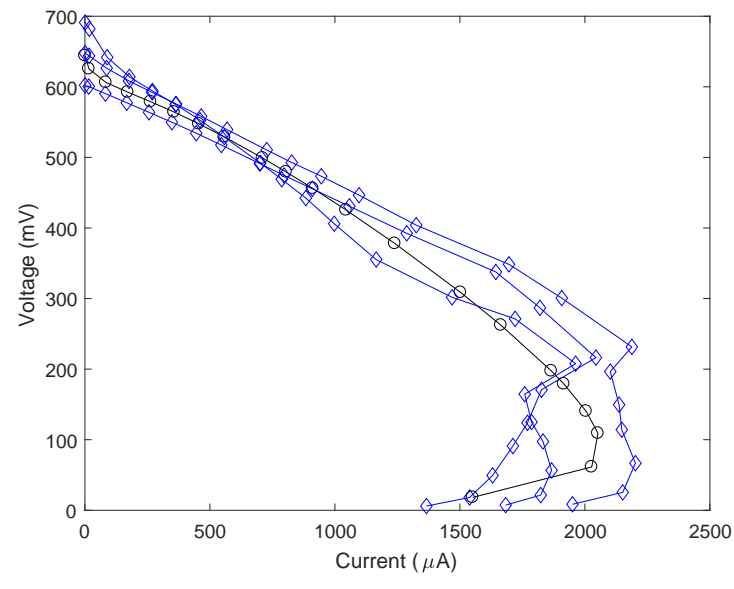

(a)

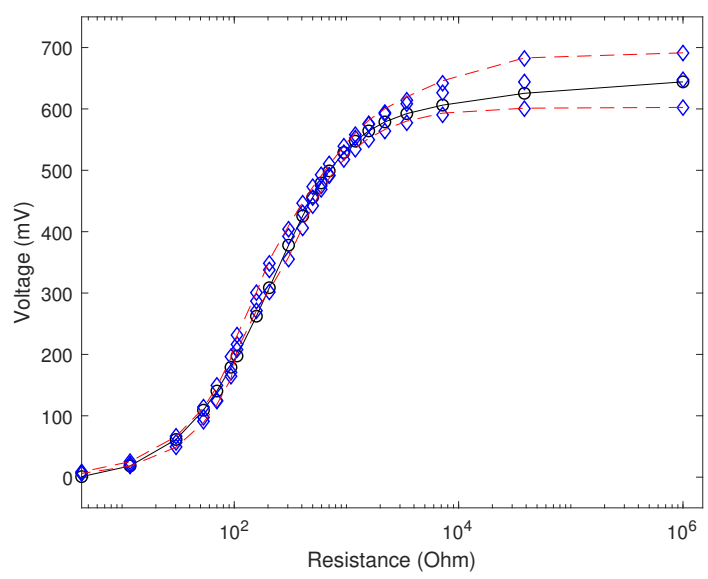

(b)

Figure 3: T_Cout type of MFCs (a) polarisation curves and (b) voltage-resistance curve, real data (in blue, diamonds), simulated data (in black, circles).

In Fig. 4 the outputs of the ANN are illustrated for the case of MFCs (T_Cin) with terracotta membrane, cathode $\left(24.5 \mathrm{~cm}^{2}\right)$ on the inside of the ceramic cylinder (at the same values of resistances used in lab).

In Fig. 5 the outputs of the ANN are shown for the case of MFCs (B_Cout) with brown terracotta membrane, cathode $\left(40 \mathrm{~cm}^{2}\right)$ on the outside of the ceramic cylinder (at the same values of resistances used in lab).

In Fig. 6 the outputs of the ANN are illustrated for the case of MFCs (B_Cin) with brown terracotta membrane, cathode $\left(28 \mathrm{~cm}^{2}\right)$ on the inside of the ceramic cylinder (at the same values of resistances used in lab).

Despite the fact that there may seem to be a higher loss of accuracy when decreasing the resistance 


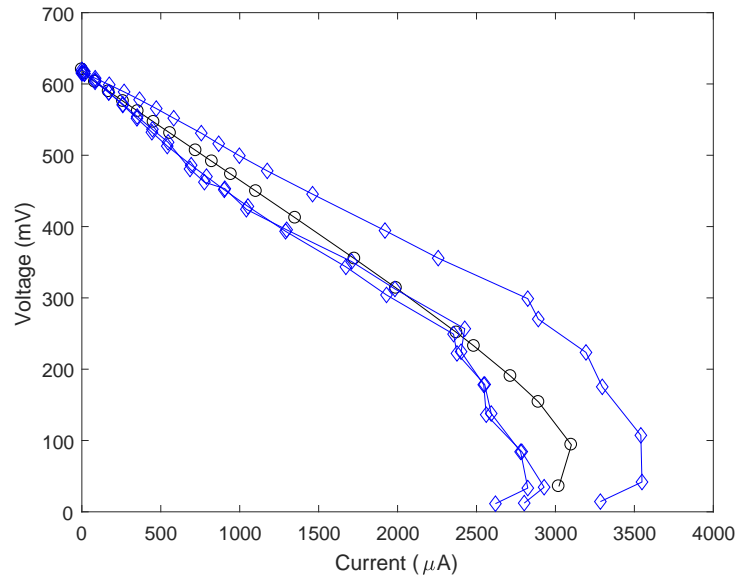

(a)

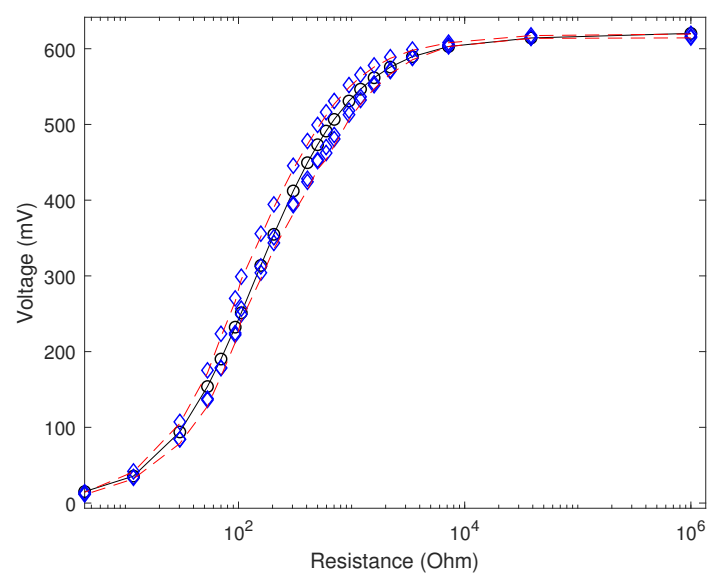

(b)

Figure 4: T_Cin type of MFCs (a) polarisation curves and (b) voltage-resistance curve, real data (in blue, diamonds), simulated data (in black, circles).

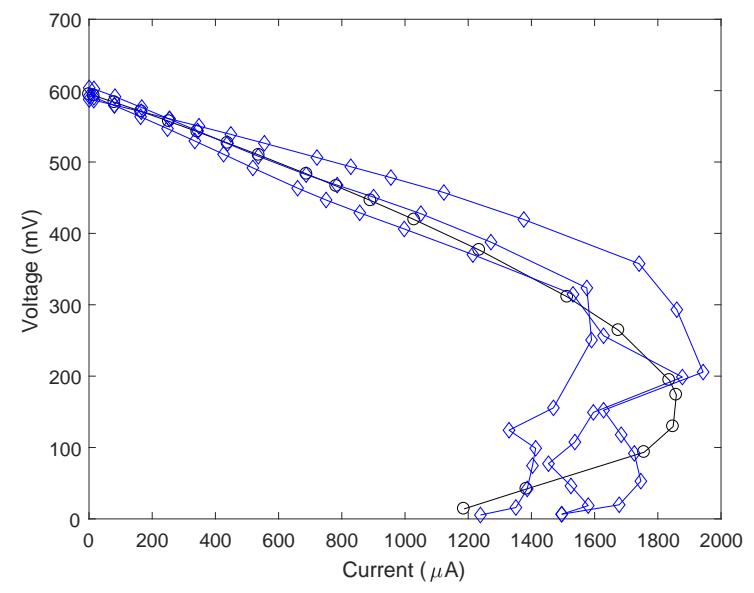

(a)

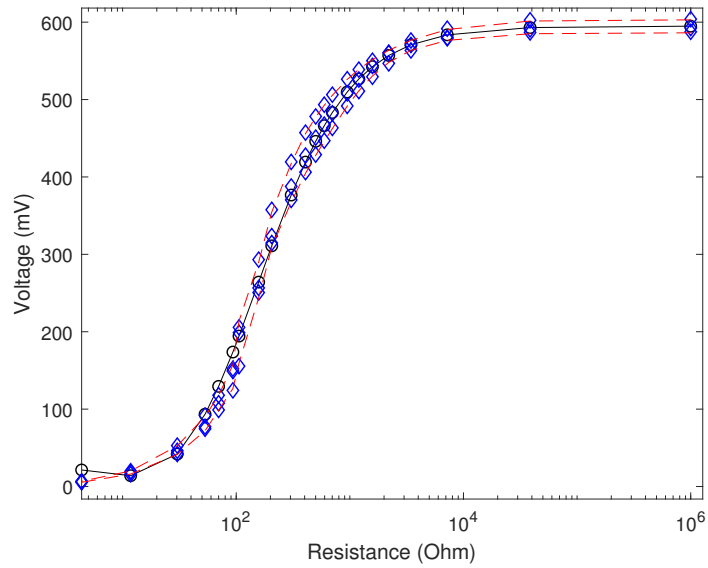

(b)

Figure 5: B_Cout type of MFCs (a) polarisation curves and (b) voltage-resistance curve, real data (in blue, diamonds), simulated data (in black, circles).

load in the polarisation curves (Figs. 36(a)), this is not true. Because the current in these curves was calculated via Ohm's law, the errors of the predicted voltage are amplified with the low values of resistance. It is apparent that there is no bias in the accuracy levels in the regression plots (Fig. 2) and the voltage to resistance plots (Figs. 3]6(b)).

The results presented in Figs. 2, 6 are derived from a single ANN with topology 4-10-1, which was trained on a randomly selected subset of the whole data-set containing the measurements of all 4 types of MFCs. In order to study the effect that the number of neurons in the hidden layer has onto the accuracy of the 


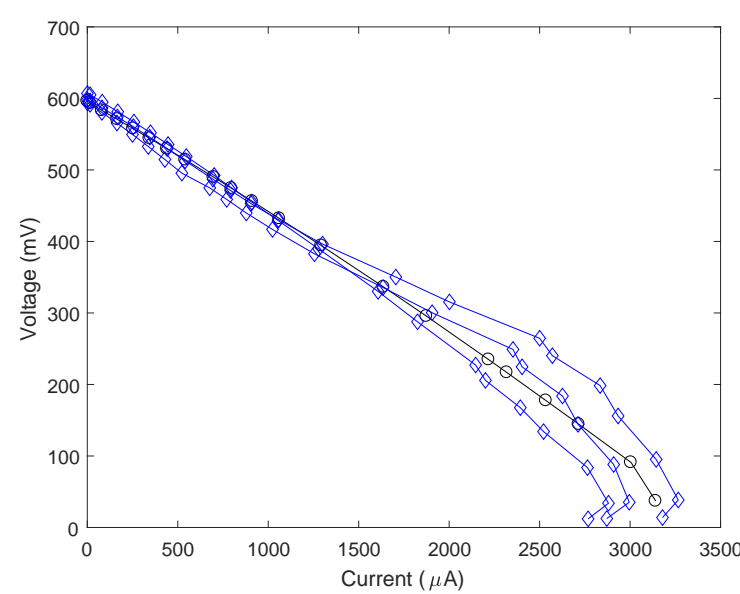

(a)

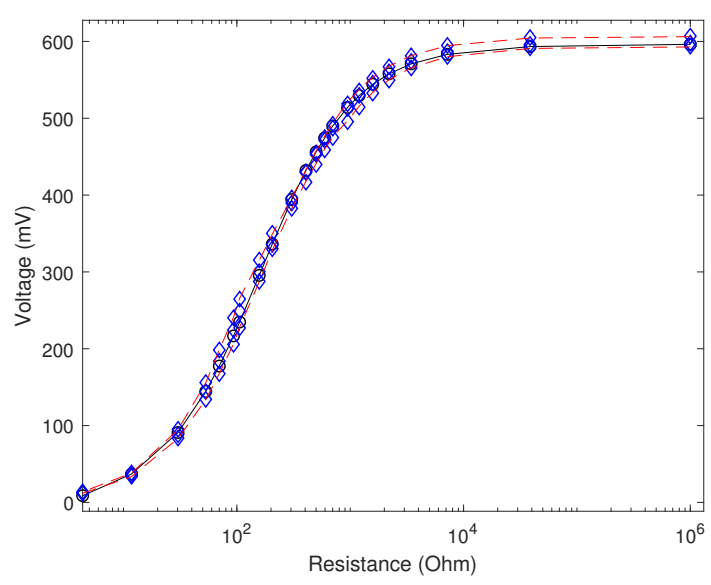

(b)

Figure 6: B_Cin type of MFCs (a) polarisation curves and (b) voltage-resistance curve, real data (in blue, diamonds), simulated data (in black, circles).

network, the following tests were executed. Several different topologies of ANNs were developed with the hidden layer neurons varying from 3 up to 15 . Each topology was tested for 10 runs to alleviate a possible impact that the initial random fragmentation of the data set (into training, validation and testing sets) has on the performance of the network. Namely, each ANN with the same topology was retrained from scratch, using every time a different training subset randomly selected from the whole set of data extracted from the 4 types of MFCs.

The distribution of correlation coefficients and mean square error of the ANNs outputs are shown in Figs. 7 and 8 , respectively. It can be observed that the topologies providing the higher performance and being less affected by the initial random fragmentation of the data set are with 10 and 15 neurons in the hidden layer. More specifically, the topology with 10 neurons, has a correlation coefficient median of 0.99683 , while the one with 15 neurons is even more efficient with a correlation coefficient median of 0.99709 .

\section{Conclusions}

Using ANNs to predict MFC outputs is becoming a popular method due to their fast implementation and their dissociation of the need for detailed knowledge of the underlying rules. When compared with rule-based models, which can provide intermediate equations and phenomena occurring within the system, ANNs have the advantage of being developed very fast and easy, but as they are trained upon specific output data, they can not provide any additional output. Nonetheless, analyzing the weights of the neurons in the network can unveil the relations of inputs and outputs. Such an analysis can reveal the relative importance of the inputs when forming the outputs [30]. 


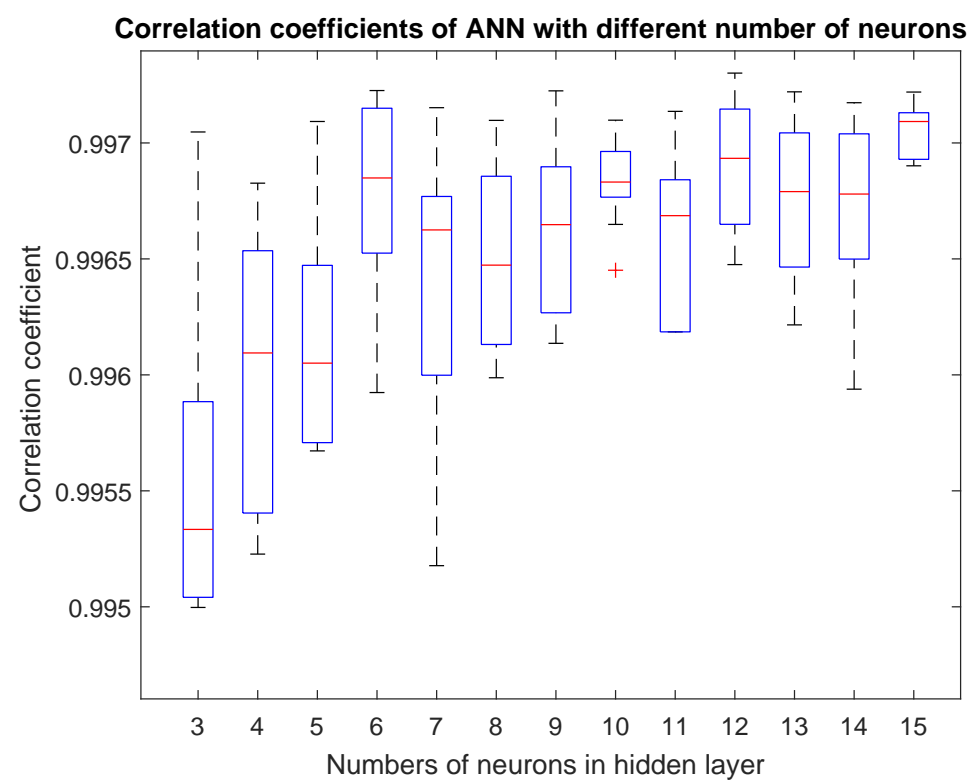

Figure 7: Distribution of correlation coefficient of the networks' outputs with the real data set, for variable numbers of neurons in the hidden layer.

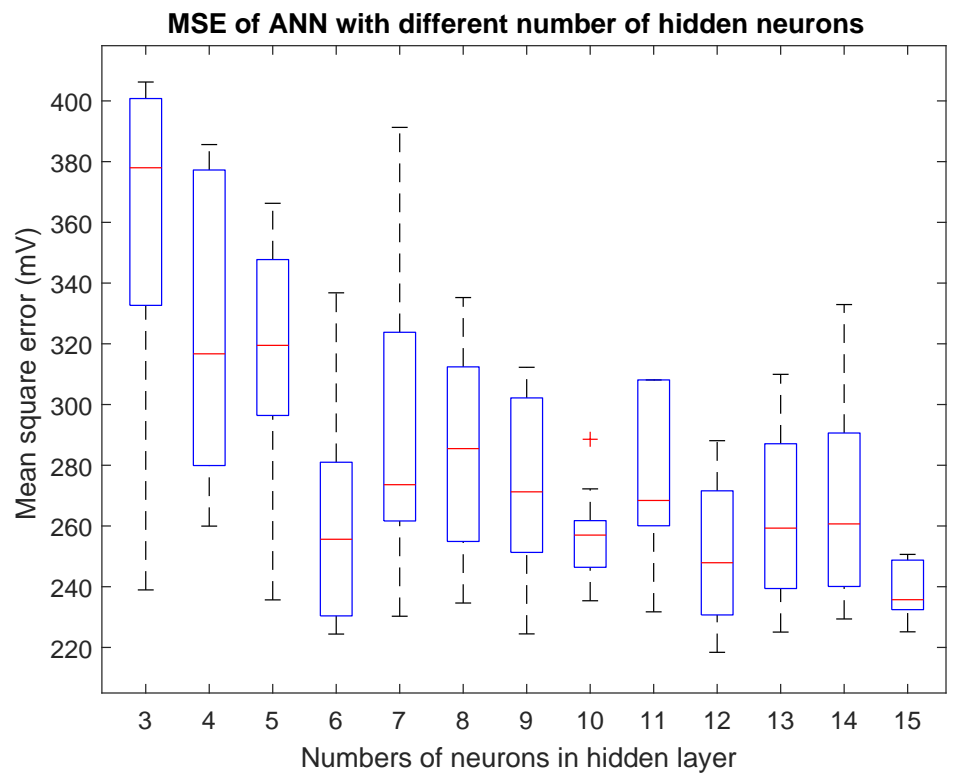

Figure 8: Distribution of mean square error of output voltage (in $\mathrm{mV}$ ) for variable numbers of neurons in the hidden layer.

In this study, an ANN was developed with a topology of 4-10-1 that predicts the voltage output of a MFC during a polarisation test. The ANN proved to be accurate in its predictions, for instance after a single training the network demonstrated a correlation coefficient $(R)$ of 0.99662 for the entire data set.

As an aspect of future work, the element of time is going to be added in the ANN, in order to predict 
the outputs of a MFC as a time series. Developing this type of ANN would be a first step towards designing an efficient fast responding controller for MFCs. As MFCs are susceptible to changes in the association of voltage and current, conventional maximum power point tracking algorithms are inadequate, particularly when voltage overshoot is observed. Thus, smarter techniques of energy harvesting control are suggested for MFCs [31 and direct methanol fuel cells 32. In particular, ANN have been successfully used as parts of power controllers in applications from photovoltaics and batteries [33, 34] to wind turbines [35] to fuel cells [36.

As shown in Figs. 3 , 4 and 5 in particular, the model was able to accurately simulate the overshoot phenomenon, which is an indication of suboptimal system performance, especially in the higher current ranges, and is an indication of ionic depletion in the anode [37, 38]. Such a phenomenon may be caused by bacterial starvation, immature biofilm, hydraulic blockage or suboptimal reactor design and these are usually elements that are not considered in modelling MFCs. To the best of the Authors' knowledge, this is the first time that such a behaviour has been replicated in an ANN model.

\section{Acknowledgements}

This work was funded by the European Union's Horizon 2020 Research and Innovation Programme under Grant Agreement No. 686585. http://livingarchitecture-h2020.eu

\section{References}

[1] V. Oliveira, M. Simes, L. Melo, A. Pinto, Overview on the developments of microbial fuel cells, Biochemical Engineering Journal 73 (2013) $53-64$.

[2] I. Ieropoulos, J. You, I. Gajda, J. Greenman, A new method for modulation, control and power boosting in microbial fuel cells, Fuel Cells 18 (2018) 663-668.

[3] A. Tremouli, J. Greenman, I. Ieropoulos, Investigation of ceramic mfc stacks for urine energy extraction, Bioelectrochemistry 123 (2018) $19-25$.

[4] C. Picioreanu, I. M. Head, K. P. Katuri, M. C. van Loosdrecht, K. Scott, A computational model for biofilm-based microbial fuel cells, Water research 41 (2007) 2921-2940.

[5] C. Picioreanu, M. van Loosdrecht, K. Katuri, K. Scott, I. Head, Mathematical model for microbial fuel cells with anodic biofilms and anaerobic digestion., Water science and technology: a journal of the International Association on Water Pollution Research 57 (2008) 965.

[6] C. Picioreanu, M. C. van Loosdrecht, T. P. Curtis, K. Scott, Model based evaluation of the effect of ph and electrode geometry on microbial fuel cell performance, Bioelectrochemistry 78 (2010) 8-24.

[7] R. Pinto, B. Srinivasan, M.-F. Manuel, B. Tartakovsky, A two-population bio-electrochemical model of a microbial fuel cell, Bioresource technology 101 (2010) 5256-5265.

[8] Y. Zeng, Y. F. Choo, B.-H. Kim, P. Wu, Modelling and simulation of two-chamber microbial fuel cell, Journal of Power Sources 195 (2010) 79-89.

[9] M.-A. Tsompanas, A. Adamatzky, I. Ieropoulos, N. Phillips, G. C. Sirakoulis, J. Greenman, Cellular non-linear network model of microbial fuel cell, Biosystems 156-157 (2017) $53-62$. 
[10] M. Tsompanas, A. Adamatzky, I. Ieropoulos, N. Phillips, G. C. Sirakoulis, J. Greenman, Modelling microbial fuel cells using lattice boltzmann methods, IEEE/ACM Transactions on Computational Biology and Bioinformatics (2018) 1-1 https://doi.org/10.1109/TCBB.2018.2831223.

[11] S. Gadkari, S. Gu, J. Sadhukhan, Towards automated design of bioelectrochemical systems: A comprehensive review of mathematical models, Chemical Engineering Journal 343 (2018) 303 - 316.

[12] B. E. Logan, J. M. Regan, Electricity-producing bacterial communities in microbial fuel cells, TRENDS in Microbiology 14 (2006) 512-518.

[13] A. Kato Marcus, C. I. Torres, B. E. Rittmann, Conduction-based modeling of the biofilm anode of a microbial fuel cell, Biotechnology and Bioengineering 98 (2007) 1171-1182.

[14] S. V. Mohan, G. Velvizhi, J. A. Modestra, S. Srikanth, Microbial fuel cell: critical factors regulating bio-catalyzed electrochemical process and recent advancements, Renewable and Sustainable Energy Reviews 40 (2014) 779-797.

[15] I. Ieropoulos, G. Pasternak, J. Greenman, Urine disinfection and in situ pathogen killing using a microbial fuel cell cascade system, PloS one 12 (2017) e0176475.

[16] G. Pasternak, J. Greenman, I. Ieropoulos, Regeneration of the power performance of cathodes affected by biofouling, Applied Energy 173 (2016) $431-437$.

[17] A. Tardast, M. Rahimnejad, G. Najafpour, K. Pirzade, N. Mokhtarian, Prediction of bioelectricity production by neural network, J Biotechnol Pharm Res 3 (2012) 62-68.

[18] A. Tardast, M. Rahimnejad, G. Najafpour, A. Ghoreyshi, G. C. Premier, G. Bakeri, S.-E. Oh, Use of artificial neural network for the prediction of bioelectricity production in a membrane less microbial fuel cell, Fuel 117 (2014) 697-703.

[19] A. Garg, V. Vijayaraghavan, S. Mahapatra, K. Tai, C. Wong, Performance evaluation of microbial fuel cell by artificial intelligence methods, Expert systems with applications 41 (2014) 1389-1399.

[20] M. Esfandyari, M. A. Fanaei, R. Gheshlaghi, M. A. Mahdavi, Neural network and neuro-fuzzy modeling to investigate the power density and columbic efficiency of microbial fuel cell, Journal of the Taiwan Institute of Chemical Engineers 58 (2016) 84-91.

[21] A. J. Jaeel, A. I. Al-wared, Z. Z. Ismail, Prediction of sustainable electricity generation in microbial fuel cell by neural network: effect of anode angle with respect to flow direction, Journal of Electroanalytical Chemistry 767 (2016) 56-62.

[22] Z. Z. Ismail, A. I. Al-wared, A. J. Jaeel, Recourse recovery of bioenergy from cellulosic material in a microbial fuel cell fed with giant reed-loaded wastewater, Biofuels (2017) 1-9.

[23] K. L. Lesnik, H. Liu, Predicting microbial fuel cell biofilm communities and bioreactor performance using artificial neural networks, Environmental science \& technology 51 (2017) 10881-10892.

[24] Y. Sewsynker, E. B. G. Kana, A. Lateef, Modelling of biohydrogen generation in microbial electrolysis cells (mecs) using a committee of artificial neural networks (anns), Biotechnology \& Biotechnological Equipment 29 (2015) 1208-1215.

[25] Y.-J. He, Z.-F. Ma, A data-driven gaussian process regression model for two-chamber microbial fuel cells, Fuel Cells 16 (2016) 365-376.

[26] J. Wang, Q. Wang, J. Zhou, X. Wang, L. Cheng, Operation space design of microbial fuel cells combined anaerobicanoxic-oxic process based on support vector regression inverse model, Engineering Applications of Artificial Intelligence 72 (2018) 340-349.

[27] A. Pinkus, Approximation theory of the mlp model in neural networks, Acta numerica 8 (1999) 143-195.

[28] I. A. Basheer, M. Hajmeer, Artificial neural networks: fundamentals, computing, design, and application, Journal of microbiological methods 43 (2000) 3-31.

[29] K. Gurney, An introduction to neural networks, CRC press, 2014.

[30] B. Mak, R. W. Blanning, An empirical measure of element contribution in neural networks, IEEE Transactions on Systems, Man, and Cybernetics, Part C (Applications and Reviews) 28 (1998) 561-564. 
[31] M. Alaraj, M. Radenkovic, J.-D. Park, Intelligent energy harvesting scheme for microbial fuel cells: Maximum power point tracking and voltage overshoot avoidance, Journal of Power Sources 342 (2017) 726-732.

[32] M. Zhang, T. Yan, J. Gu, Maximum power point tracking control of direct methanol fuel cells, Journal of Power Sources 247 (2014) 1005-1010.

[33] L. M. Elobaid, A. K. Abdelsalam, E. E. Zakzouk, Artificial neural network-based photovoltaic maximum power point tracking techniques: a survey, IET Renewable Power Generation 9 (2015) 1043-1063.

[34] C. Sun, F. Sun, S. J. Moura, Nonlinear predictive energy management of residential buildings with photovoltaics \& batteries, Journal of Power Sources 325 (2016) $723-731$.

[35] Hui Li, K. L. Shi, P. G. McLaren, Neural-network-based sensorless maximum wind energy capture with compensated power coefficient, IEEE Transactions on Industry Applications 41 (2005) 1548-1556.

[36] A. Harrag, H. Bahri, Novel neural network ic-based variable step size fuel cell mppt controller: Performance, efficiency and lifetime improvement, International Journal of Hydrogen Energy 42 (2017) 3549 - 3563.

[37] J. Winfield, I. Ieropoulos, J. Greenman, J. Dennis, The overshoot phenomenon as a function of internal resistance in microbial fuel cells, Bioelectrochemistry (Amsterdam, Netherlands) 81 (2011) 22-27.

[38] J. Winfield, I. Ieropoulos, J. Greenman, The overshoot phenomenon as a function of internal resistance in microbial fuel cells, Journal of Biotechnology 150 (2010) 23. 